\title{
A Common Founder Mutation in the EDA-A1 Gene in X-Linked Hypodontia
}

\author{
Mazen Kurban ${ }^{\mathrm{a}}$ Eleni Michailidis ${ }^{\mathrm{b}}$ Muhammad Wajid $^{\mathrm{a}}$ Yutaka Shimomura $^{\mathrm{a}}$ \\ Angela M. Christiano ${ }^{\mathrm{a}, \mathrm{c}}$ \\ ${ }^{\text {a } D e p a r t m e n t ~ o f ~ D e r m a t o l o g y, ~}{ }^{\mathrm{b}} \mathrm{School}$ of Dental Medicine and ${ }^{\mathrm{C}}$ Department of Genetics and Development, \\ Columbia University, New York, N.Y., USA
}

\section{Key Words}

X-linked recessive hypohidrotic ectodermal dysplasia •

$X$-linked recessive hypodontia $\cdot E D A-A 1$ gene

\begin{abstract}
Background: X-linked recessive hypohidrotic ectodermal dysplasia (XLHED; OMIM 305100) is a rare genodermatosis characterized clinically by developmental abnormalities affecting the teeth, hair and sweat glands. Mutations in the $E D A-A 1$ gene have been associated with XLHED. Recently, mutations in the EDA-A1 gene have also been implicated in isolated X-linked recessive hypodontia (XLRH; OMIM 313500). Methods: We analyzed the DNA from members of 3 unrelated Pakistani families with $\mathrm{XLRH}$ for mutations in the EDA-A1 gene through direct sequencing and performed haplotype analysis. Results: We identified a common missense mutation in both families designated c.1091T $\rightarrow C$ (p.M364T). Haplotype analysis revealed that this is a founder mutation in the 3 families. Conclusion: XLHED is a syndrome with variable clinical presentations that contain a spectrum of findings, including hypodontia. We suggest that XLRH should be grouped under XLHED as both share several phenotypic and genotypic similarities.
\end{abstract}

Copyright $\odot 2010$ S. Karger AG, Basel

\section{Introduction}

Ectodermal dysplasias (EDs) are a group of disorders characterized by developmental abnormalities in at least 2 of the following 4 structures: hair, teeth, nails and sweat glands. To be classified as ED, a syndrome is required to have abnormalities in at least 2 of the 4 listed tissues. Hypohidrotic ectodermal dysplasia (HED), the most common type of EDs, is inherited most commonly as an Xlinked recessive disease but can also be inherited in autosomal dominant and recessive patterns [1-3]. Clinically, patients may be born with a collodion membrane. Hair abnormalities can range from mild hair loss to a complete absence of hair. Frequently, when the hair is present, it is usually hypopigmented but tends to darken around puberty. In addition to the hair abnormalities, patients have failed development of the eccrine sweat glands, which is also variable among the patients. A characteristic presentation includes repeated episodes of elevated body temperature that can be life-threatening because of the inability to sweat $[4,5]$.

Hypodontia/oligodontia is a universal feature among HEDs and may be associated with peg-shaped teeth. HED patients have a characteristic facies which includes frontal bossing, absent or scarce eyebrows, saddle nose,

\section{KARGER}

(C) 2010 S. Karger AG, Basel

Fax +41613061234 E-Mail karger@karger.ch www.karger.com www.karger.com/drm
Angela M. Christiano, $\mathrm{PhD}$

Department of Dermatology, Columbia University

Russ Berrie Medical Center, 1150 St. Nicholas Avenue

New York, NY 10032 (USA)

Tel. +1 212851 4850, Fax +1 212851 4810,E-Mail amc65@ columbia.edu 
periorbital wrinkling and hyperpigmentation, sebaceous gland hyperplasia, an uncommon finding possibly compensating for hypohidrosis, and thickened, everted lips. Some patients may exhibit thickened viscous nasal and respiratory tract secretions with recurrent infections, suggesting that secretory glands in addition to the eccrine sweat glands are also affected $[4,5]$.

The molecular basis of X-linked hypohidrotic ectodermal dysplasia (XLHED) involves disruption in ectodysplasin A (EDA). EDA is a member of the tumor necrosis factor (TNF) family and is a type II transmembrane protein [6]. EDA consists of 3 regions: an $\mathrm{N}$-terminal intracellular domain, a transmembrane domain and an extracellular domain as well as a C-terminal domain which possesses the TNF homology domain. In order to be functionally active, EDA should be cleaved and released out of the cells where it forms a trimer that binds to the EDA receptor (EDAR) and activates it. EDA is cleaved at a special site referred to as the furin cleavage site; thus, any mutation at this site will result in the inability to form the active EDA trimer resulting in disease [6]. EDA is comprised of 8 exons, and several isoforms exist due to alternative splicing. The 2 most common isoforms are EDA-A1 and EDA-A2, which only differ by 2 amino acids, with EDA-A1 consisting of 391 amino acids while EDA-A2 consists of 389 amino acids. These 2 amino acids confer significant differences in function $[7,8]$. Mutations in the EDA-A1 isoform are implicated in the pathogenesis of XLHED. EDA-A1 binds to the EDAR, which interacts with the EDAR-associated death domain, leading to the activation of nuclear factor $\kappa \mathrm{B}$ that translocates into the nucleus and activates other transcription factors involved in the development of skin appendages and teeth $[3,5]$. Therefore, mutations in any gene along the pathway would be expected to result in HED; however to date, mutations have been detected mainly in EDA-A1 leading to XLHED, and less commonly in the EDAR and EDAR-associated death domain genes, leading to either autosomal recessive HED or autosomal dominant HED. Moreover, mutations in the nuclear factor $\kappa \mathrm{B}$ inhibitor I $\mathrm{B} \alpha$ and in NEMO (IKK $\gamma$ ) cause a human HED syndrome with immunodeficiency [9].

Recently, there have been several reports of mutations in the EDA-A1 gene leading to an isolated form of $\mathrm{X}$ linked recessive hypodontia (XLRH) [7, 10-12]. This observation was somewhat surprising, since perturbation of the EDA-A1 pathway is also implicated in the development of hair and sweat glands.
Here, we report 3 Pakistani families with a common founder mutation in the $E D A-A 1$ gene, presenting clinically with isolated hypodontia.

\section{Materials and Methods}

DNA Extraction

After obtaining informed consent, we collected peripheral blood samples from family members in EDTA-containing tubes under institutional approval and in adherence to the Declaration of Helsinki principles. Genomic DNA was isolated from these samples according to standard techniques.

\section{Mutation Analysis}

All exons of the EDA-A1 gene with adjacent sequences of exonintron borders were amplified by PCR with primers and conditions described previously [4]. The amplified PCR products were directly sequenced in an ABI Prism 310 Automated Sequencer, using the ABI Prism Big Dye Terminator Cycle Sequencing Ready Reaction Kit (PE Applied Biosystems).

\section{Haplotype Analysis}

We performed haplotype analysis using 4 microsatellite markers (D15S162, DXS8031, DXS8107 and DXS8052) spanning the $E D A-A 1$ gene. The amplification conditions for each PCR were $94^{\circ} \mathrm{C}$ for $2 \mathrm{~min}$, followed by 35 cycles of $94^{\circ} \mathrm{C}$ for $30 \mathrm{~s}, 55^{\circ} \mathrm{C}$ for $30 \mathrm{~s}$ and $72^{\circ} \mathrm{C}$ for $30 \mathrm{~s}$, with a final extension at $72^{\circ} \mathrm{C}$ for $7 \mathrm{~min}$. The amplified PCR products were run on an $8 \%$ polyacrylamide gel, and genotyping was performed by visual inspection.

\section{Results}

\section{Patients}

The 3 families originated from different regions in Pakistan and are completely unrelated. The inheritance in the 3 families, family A, family B and family C, was consistent with an X-linked recessive pattern (fig. 1a). By history, patients reported having several absent teeth as long as they could recall. Patients reported normal sweating and no recurrent episodes of elevated body temperature. On physical examination there was no gross craniofacial deformity except for thickened and everted lips in some of the patients (fig. 1b). Dental abnormalities were variable among members of the 3 families, but the common finding in all patients was the absence of mandibular central incisors (fig. 1b). Members of family A had severer dental abnormalities, including loss of mandibular and maxillary lateral incisors, canines and first and second premolars (fig. 1b). Interestingly, some patients from the 3 families also had mild hypotrichosis (fig. 1c). 


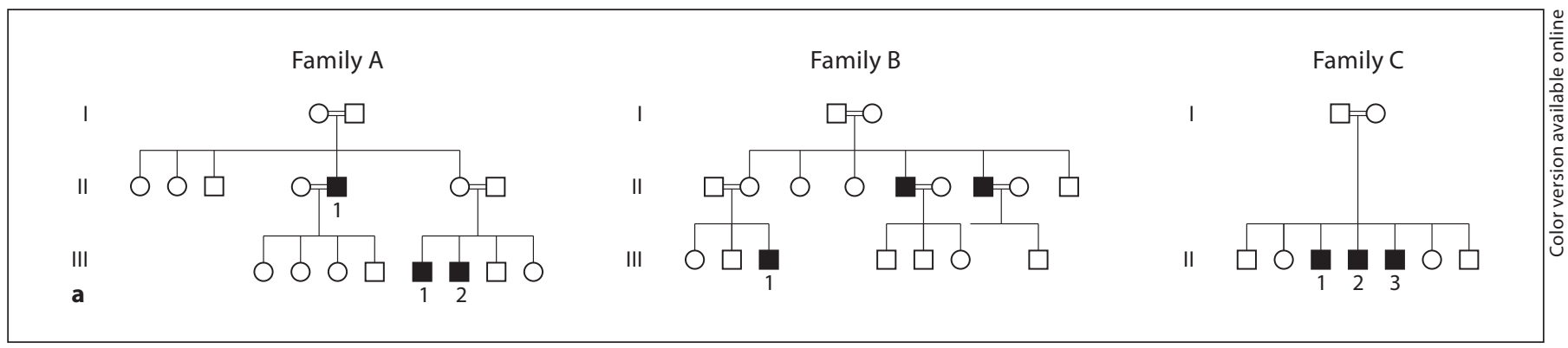

Fig. 1. a Three families with XLRH showing an X-linked recessive pattern of inheritance. b An individual with thickened and everted lips, a common finding in XLHED. The most common tooth abnormality in patients from the 3 families was absent central mandibular incisors. A member from family A showing severe dental abnormalities, characterized by bilateral absence of the central and lateral mandibular incisors, the canine tooth, the 2 premolars and the first molar. c An individual with XLRH showing a mild form of hypotrichosis.
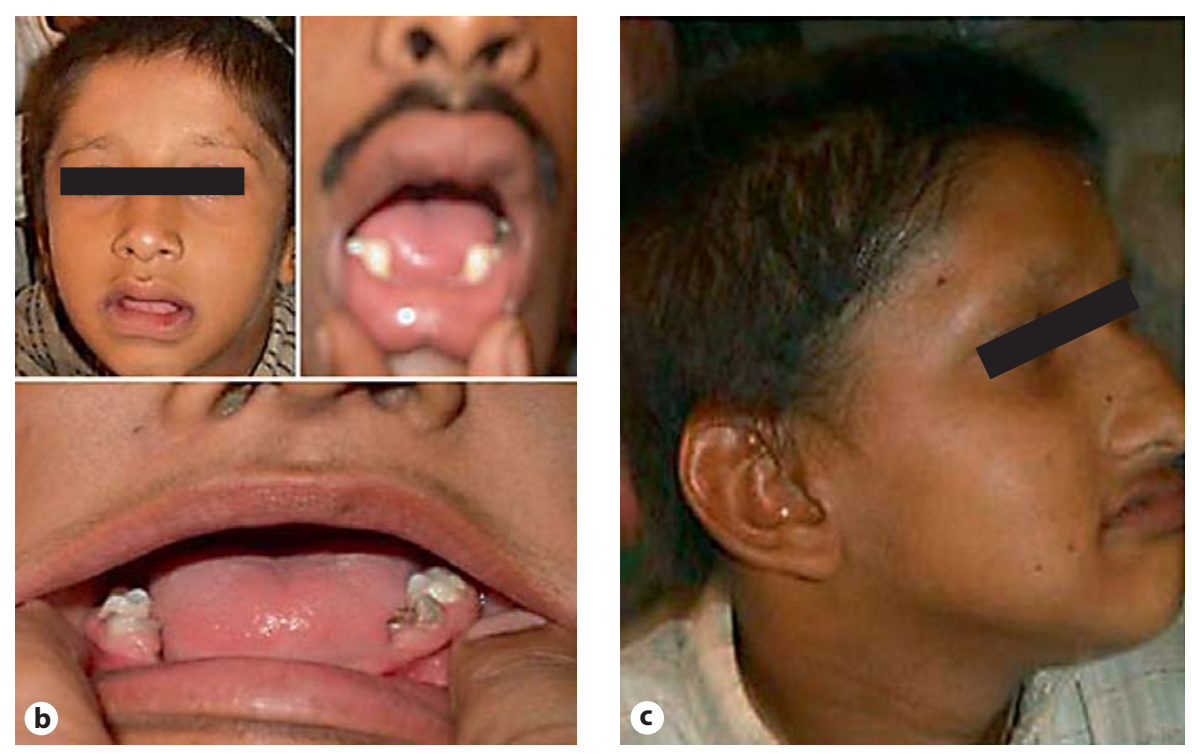

\section{Genetic Analysis}

Direct sequencing of the EDA-A1 gene in affected individuals of the 3 families revealed the same missense mutation, designated c.1091T $\rightarrow$ C (p.M364T; fig. 2a). Microsatellite marker analysis showed a shared haplotype among the affected members of both families, suggesting a common founder mutation (fig. 2b).

\section{Discussion}

To date, 4 genes known to be implicated in tooth development, $P A X 9, M S X 1, A X I N 2$ and $E D A-A 1$, have been well characterized [13]. Two main differences exist between the phenotypes arising from EDA-A1 mutations and the disorders arising from mutations in the other 3 genes. The first difference is that $E D A-A 1$ mutations are inherited in an X-linked recessive pattern, while the others are inherited as autosomal dominant conditions [13]. The second distinction is related to the clinical presentation. EDA-A1 mutations lead to abnormal tooth develop- ment, predominantly affecting the mandibular teeth, whereas the other 3 genes primarily affect development in the maxillary region; moreover, in EDA-1 the anterior teeth are predominantly affected while in the others it is predominantly the posterior teeth. The average number of missing teeth in XLHED is 3.50 in the maxillary region and 5.30 in the mandibular region $[13,14]$.

The most common teeth involved are the mandibular and maxillary lateral incisors and the mandibular central incisors [13]. EDA is expressed early in the development of the teeth, starting at the bud stage, and is then continuously expressed in the mesenchyme until the end of the cap stage [15]. This underscores the importance of the $E D A$ gene not only in the formation of the normal number of teeth, but also in their morphology.

In recent years, there have been several reports of mutations in the EDA-A1 gene resulting in isolated $\mathrm{X}$ linked hypodontia. The mutations in EDA-A1 causing isolated X-linked hypodontia are V365A, Q358E, D316G, T338M, M364T, G255C, G291R, A259E, R289C, Arg334His and S374R [7, 10-12, 16, 17]. It is noteworthy 
Fig. 2. a $A$ missense mutation designated c.1091T $\rightarrow$ C (M364T) in affected individuals from the 3 families. b Haplotype analysis revealed that the mutation M364T is a founder mutation in the Pakistani population.

$$
\text { G T CA A } \frac{364 T}{A C G} \text { G T G AC }
$$$$
\text { G T C A A } \frac{364 M}{A T G} \text { G T G A C }
$$
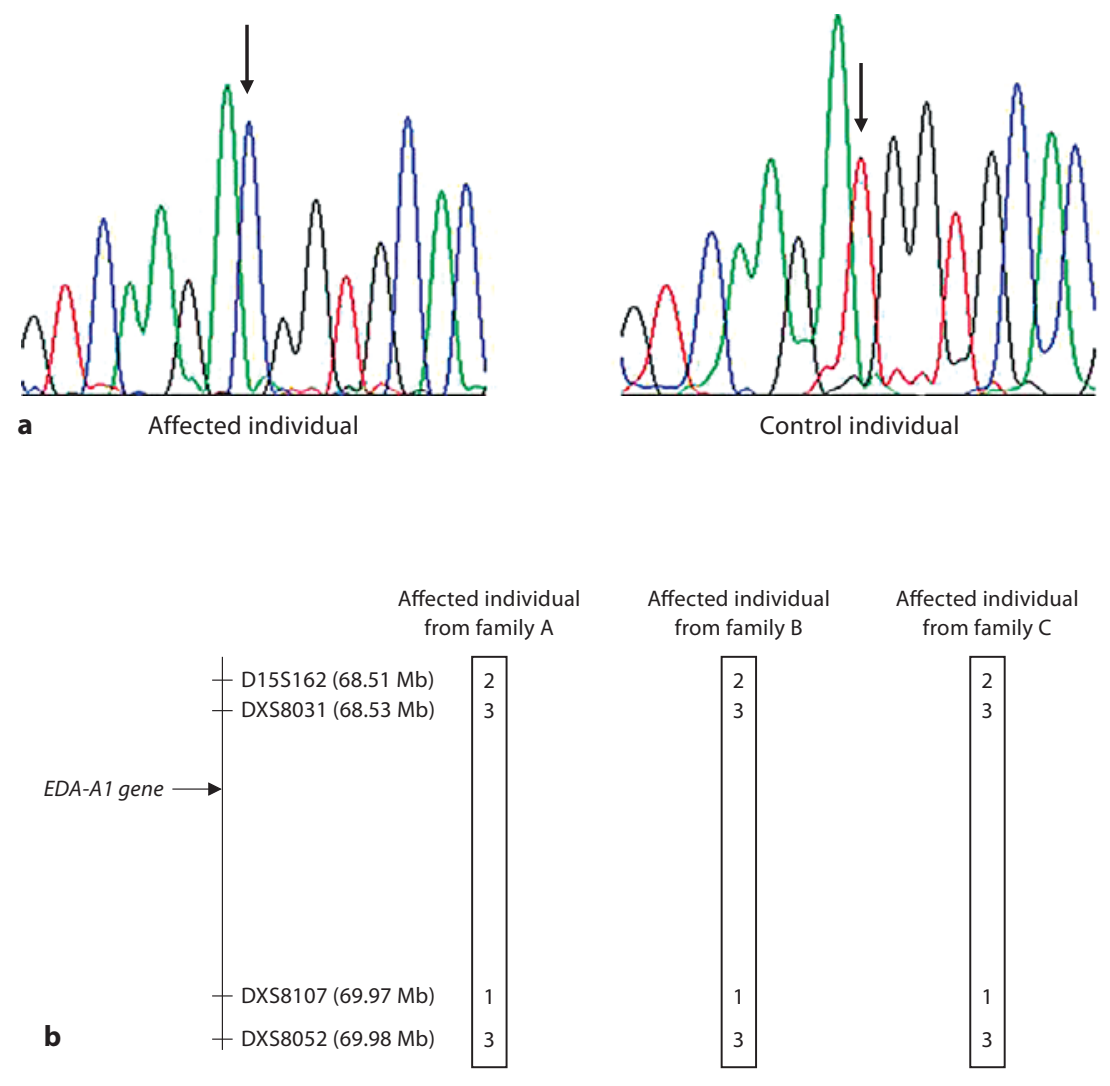

that most mutations causing isolated XLRH are missense mutations that lie within the TNF homology domain. Normally, the TNF homology domain (residues 245-391) forms a homotrimer [18] which is essential for the correct binding of EDA-A1 to EDAR. Functional studies have shown that XLHED-causing mutations result in loss of binding of EDA-A1 to its receptor [16] while tooth agenesis-causing mutations do not inhibit the binding completely, since residual binding is observed [7]. Thus, the XLRH mutations still possess some EDA/EDAR signaling which may be sufficient for the development of other appendageal structures, but insufficient for complete tooth development [7]. In addition, generally patients with isolated XLRH have less severe dental anomalies than patients with XLHED, providing additional evidence that some EDA/EDAR signaling is partially active but not enough to form complete and normal teeth [7].
Although residual signaling of EDA/EDAR in patients with isolated XLRH may be sufficient for the normal development of the hair follicles and sweat glands, a subclinical involvement of these appendageal structures may still exist. Skin biopsies confirming the presence of sweat glands may not be definitive, since these eccrine sweat glands might not be $100 \%$ functional, and a starch iodine test may be helpful in quantitating sweating. Moreover, mild hair abnormalities might not be clinically apparent and observation under light and electron microscope may be helpful. XLHED and XLRH share several features, they both have mutations in the EDA-A1 gene involved in the same signaling pathway and are transmitted as X-linked recessive diseases. Both conditions are associated with dental abnormalities, and as we have shown here, hypotrichosis can be observed in some patients with XLRH. XLHED patients will show clinically abnormal sweating and severer hair abnormalities as mutations in 
XLHED will completely inhibit the EDA/EDAR signaling, whereas in XLRH, residual activity may still be present. Given the striking similarities, we postulate that XLHED and XLRH represent a spectrum of one disease, and XLRH should be included under the same grouping as XLHED.

Here, we studied 3 unrelated families from Pakistan with a missense mutation, M364T, in the EDA-A1 gene showing clinical features of XLHED limited mainly to hypodontia. Haplotype analysis was consistent with a common founder ancestor in the Pakistani population. Although the main clinical presentation was hypodontia, the majority of patients had thickening and eversion of the lips, and few had mild hypotrichosis, which are com- mon findings in XLHED. Therefore, close examination of patients with mutations suggested to be related to isolated hypodontia may, in fact, reveal mild features of XLHED, suggesting that XLHED and XLRH represent variable expressions within the same disease spectrum.

\section{Acknowledgements}

We gratefully acknowledge the families for having participated in this study. We also thank Helen Lam for expert technical assistance. This study was supported in part by a Research Career Development Award from the Dermatology Foundation (to Y.S.) and an NIH grant from USPHS, NIH/NIAMS RO1 AR44924 (to A.M.C.).

\section{References}

1 Bayes M, Hartung AJ, Ezer S, et al: The anhidrotic ectodermal dysplasia gene (EDA) undergoes alternative splicing and encodes ectodysplasin-A with deletion mutations in collagenous repeats. Hum Mol Genet 1998;7: 1661-1669.

2 Monreal AW, Ferguson BM, Headon DJ, Street SL, Overbeek PA, Zonana J: Mutations in the human homologue of mouse dl cause autosomal recessive and dominant hypohidrotic ectodermal dysplasia. Nat Genet 1999; 22:366-369.

$\checkmark 3$ Headon DJ, Emmal SA, Ferguson BM, et al: Gene defect in ectodermal dysplasia implicates a death domain adapter in development. Nature 2001;414:913-916.

$\checkmark 4$ Kere J, Srivastava AK, Montonen O, et al: $\mathrm{X}$-linked anhidrotic (hypohidrotic) ectodermal dysplasia is caused by mutation in a novel transmembrane protein. Nat Genet 1996; 13:409-416.

5 Mikkola ML, Thesleff I: Ectodysplasin signaling in development. Cytokine Growth Factor Rev 2003;14:211-224.

6 Elomaa O, Pulkkinen K, Hannelius U, Mikkola M, Saarialho-Kere U, Kere J: Ectodysplasin is released by proteolytic shedding and binds to the EDAR protein. Hum Mol Genet 2001;10:953-962.
7 Mues G, Tardivel A, Willen L, et al: Functional analysis of ectodysplasin-A mutations causing selective tooth agenesis. Eur J Hum Genet 2010;18:19-25.

8 Yan M, Wang LC, Hymowitz SG, et al: Twoamino acid molecular switch in an epithelial morphogen that regulates binding to two distinct receptors. Science 2000;290:523527.

9 Mikkola ML: Molecular aspects of hypohidrotic ectodermal dysplasia. Am J Med Genet A 2009;149A:2031-2036.

10 Li S, Li J, Cheng J, et al: Non-syndromic tooth agenesis in two Chinese families associated with novel missense mutation in the TNF domain of EDA (ectodysplasin A). PLoS One 2008;3:e2396.

11 Rasool M, Schuster J, Aslam M, et al: A novel missense mutation in the EDA gene associated with $\mathrm{X}$-linked recessive isolated hy podontia. J Hum Genet 2008;53:894-898.

12 Fan H, Ye X, Shi L, et al: Mutations in the EDA gene are responsible for X-linked hypohidrotic ectodermal dysplasia and hypodontia in Chinese kindreds. Eur J Oral Sci 2008; 116:412-417.

13 Han D, Gong Y, Wu H, et al: Novel EDA mutation resulting in $\mathrm{X}$-linked non-syndromic hypodontia and the pattern of EDA-associated isolated tooth agenesis. Eur J Med Genet 2008;51:536-546.
14 Barbería E, Saavedra D, Arenas M, Maroto M: Multiple agenesis and anhidrotic ectodermal dysplasia: a comparative longitudinal study of dental similarities and genetic differences in two groups of children. Eur J Paediatr Dent 2006;7:113-121.

15 Tucker AS, Headon DJ, Schneider P, et al: Edar/Eda interactions regulate enamel knot formation in tooth morphogenesis. Development 2000;127:4691-4700.

16 Schneider P, Street SL, Gaide O, et al: Mutations leading to X-linked hypohidrotic ectodermal dysplasia affect three major functional domains in the tumor necrosis factor family member ectodysplasin-A. J Biol Chem 2001;276:18819-18827.

17 Song S, Han D, Qu H, et al: EDA gene mutations underlie non-syndromic oligodontia. J Dent Res 2009;88:126-131.

18 Ezer S, Bayés M, Elomaa O, Schlessinger D, Kere J: Ectodysplasin is a collagenous trimeric type II membrane protein with a tumor necrosis factor-like domain and co-localizes with cytoskeletal structures at lateral and apical surfaces of cells. Hum Mol Genet 1999;8:2079-2086. 